\title{
The role of microscopy in the diagnosis of sexually transmitted infections in women
}

\author{
Hilary Andrews, Nigel Acheson, Mia Huengsberg, Keith W Radcliffe
}

\begin{abstract}
Introduction-Microscopy of genital secretions is routinely performed in female patients attending genitourinary medicine clinics. It diagnoses only $50-70 \%$ of gonorrhoea, $40-80 \%$ of trichomoniasis and has no value in the diagnosis of chlamydial infection. This study was therefore conducted to reassess the role of routine microscopy in female patients.

Subjects and Methods-One thousand consecutive women attending the genitourinary medicine clinic of the General Hospital, Birmingham, were studied prospectively. The first 500 women had routine microscopy performed. The second 500 women had microscopy performed only if they complained of symptoms, were known gonorrhoea contacts, or when an abnormal vaginal discharge was noted by the examining clinician.
\end{abstract}

Results-In the routine microscopy group, $46(9 \cdot 2 \%)$ women had gonorrhea; 30 of these were diagnosed by microscopy and subsequently confirmed on culture and 16 by culture alone; of these, two (4.3\%) defaulted from followup and were not treated. In the selective microscopy group 139 women (28\%) did not require microscopy. Thirty three women had positive culture for Neisseria gonorrhoeae. Of these, seven were diagnosed by microscopy, the rest by culture alone. All patients were successfully treated. No patients with trichomoniasis in the routine microscopy group and only two $(4 \cdot 3 \%)$ in the selective microscopy group were lost to follow-up.

Conclusion-In this study, the selective policy in the second group led to a significant reduction in microscopy. Such a policy has the benefits of saving time for patients and staff, more efficient utilisation of manpower and resources. It did not lead to any significant delay in the diagnosis and treatment of patients with sexually transmitted infections.

(Genitourin Med 1994;70:118-120)

\section{Introduction}

Microscopy of the genital secretions is routinely done in women attending genitourinary medicine (GUM) clinics as it is capable of leading to an immediate diagnosis of gonorrhoea, trichomoniasis, candidosis and bacterial vaginosis. The standard method of diagnosing gonorrhoea is by visualising Gram-negative intracellular diplococci under the microscope. This is extremely successful in diagnosing urethral gonorrhoea in males, having a sensitivity of approximately $90 \%$ $95 \%,{ }^{1-5}$ but is much less useful for the diagnosis of gonorrhoea in female patients as the sensitivity of microscopy for detecting Neisseria gonorrhoeae from the cervix is only $50 \%-70 \%^{6-10}$. Therefore microbiological culture is of crucial importance for the diagnosis of gonorrhoea in women.

Trichomonas vaginalis is sought on a wet mount preparation where the individual flagella and undulating membrane are easily recognisable. Unfortunately the wet mount reveals Trichomonas vaginalis in only $40-80 \%$ of cases $^{11-14}$ and cultures must always be performed for confirmation.

Routine use of microscopy as described above allows a diagnosis to be made before the woman leaves the clinic in a proportion of cases. This means that treatment and the tracing of sexual contacts can be carried out immediately and the importance of follow-up explained to the patient. However, it does have the disadvantage of requiring patients to wait for their microscopy results. It also makes considerable demands on clinic staff who have to stain and examine the slides and then spend time conveying and explaining the results to their patients. In addition there is the risk of a woman being falsely reassured that she is free from sexually transmitted infection by the negative microscopy results. As discussed above, the sensitivity of microscopy for the diagnosis of gonorrhoea and trichomoniasis falls significantly short of $100 \%$ and microscopy is completely without value for the immediate diagnosis of the common and serious sexually transmitted pathogen, Chlamydia trachomatis.

This study was therefore conducted to reassess the role of immediate routine microscopy of genital secretions in the management of sexually transmitted diseases in women.

Subjects and methods

Between January and September 1992, 1,000 women attending the Whittall Street Genitourinary Medicine Clinic at the General Hospital, Birmingham for the first time were
Address correspondence to: Dr H Andrews

Accepted for publication 4 November 1993 
studied. All women in the study had intact genital tracts. The patients studied attended consecutively and were divided into two groups as follows:

\section{1) Universal microscopy group}

Five hundred women had routine microscopy of Gram-stained exudates from the urethra, vagina and endo-cervix, microscopy of a vaginal wet preparation, measurement of vaginal $\mathrm{pH}$ and an amine test. Cultures for Neisseria gonorrhoeae onto selective media containing vancomycin, colistin, nystatin and trimethoprim (VCNT) were taken from the urethra, high vagina, endo-cervix and rectum Culture for Trichomonas vaginalis was performed on vaginal secretions. An endocervical swab was taken and tested for Chlamydia trachomatis by an ELISA method.

\section{2) Selective microscopy group}

The second consecutive 500 women had microscopy only if: (1) they spontaneously complained of one or more of the following: vaginal discharge; vulval or vaginal soreness; vulval itching; lower abdominal pain; deep dyspareunia or superficial dysuria; (2) an abnormal vaginal discharge was observed by the examining clinician; (3) the patient was a contact of a patient known to have gonorrhoea. All patients had cultures taken as in the first group. The slides were interpreted by Medical Laboratory Scientific Officers (MLSO) rotating to the Department from the Microbiology Department of the General Hospital, Birmingham.

In accordance with standard departmental practice all women with gonorrhoea were personally interviewed by a health adviser in order to carry out contact tracing. In the selective microscopy group, detailed histories were taken by the health advisers to exclude the possibility of further sexual intercourse while waiting for culture results.

The following results concern the diagnosis of gonorrhoea and trichomoniasis which are the two sexually transmitted infections detectable by microscopy.

\section{Results}

1) Universal microscopy group

In the first 500 women, routine microscopy was performed on all patients. The mean age of the women was 26 years (range 15-58 years).

(i) Gonorrhoea: 46 of the 500 women (9.2\%) had cultures positive for Neisseria gonorrhoeae. Thirty of these 46 women (65\%) had their gonorrhoea diagnosed by microscopy and were informed of this immediately. Sixteen of the 46 women (35\%) were diagnosed by culture alone; treatment and contact-tracing were successfully carried out in fourteen of them. Two of the 46 women $(4 \cdot 3 \%)$ were lost to follow-up.

(ii) Trichomonas vaginalis: 16 of the 500 women $(3 \cdot 2 \%)$ were positive for Trichomonas vaginalis on culture. Fifteen of these patients $(94 \%)$ were diagnosed by microscopy and received treatment immediately. One patient (6\%) was diagnosed by culture alone. No patients were lost to follow-up.

\section{2) Selective microscopy group}

In the 500 women where microscopy was performed on the selective criteria outlined above there were four protocol failures, leaving 496 women suitable for analysis. The mean age in this group was 26.5 years (range 14-68 years). In 139 women (28\%) microscopy was not performed.

(i) Gonorrhoea: Thirty three of the 496 women $(6 \cdot 7 \%)$ had positive culture for Neisseria gonorrhoeae. Microscopy was done in 32 of these 33 cases (97\%), but only seven (22\%) were diagnosed by microscopy. Twenty six ( $79 \%)$ were diagnosed by culture only. All of the patients were recalled, successfully treated and contact-traced.

(ii) Trichomonas vaginalis: Sixteen of the 496 women (3.2\%) grew Trichomonas vaginalis on culture. Microscopy was done in all of these 16 patients but Trichomonas vaginalis was diagnosed in only $9(56 \%)$ who were treated immediately. Two of the seven patients $(12.5 \%)$ diagnosed on culture only were lost to follow-up.

\section{Discussion}

When this policy of selective microscopy was carried out, $28 \%$ of new female patients did not require microscopy. This reduction in microscopy has obvious benefits. It saves time for the patients, who do not have to wait for microscopy results and be interviewed by the doctor on a second occasion. It saves time for the staff. The examination time for the doctor and nurse is shorter. The MLSO has fewer slides to read and should therefore be able to spend more time on the slides that they do see. Alternatively more patients can be fitted into any one clinic. It is interesting that the MLSOs in the first (universal microscopy) group diagnosed $65 \%$ cases of gonorrhoea on initial microscopy, whereas in the second (selective microscopy) group only $21.8 \%$ of the gonorrhoea cases were so diagnosed. This may be a reflection of changes in personnel; during the study period, new MLSOs were being trained in the department.

Also of importance are the savings made in equipment such as slides and reagents if $28 \%$ less microscopy is performed. Another benefit of this policy of selective microscopy is that it prevents the patient from being falsely reassured that she has no disease at all or that she only has candidiasis or bacterial vaginosis. In the universal microscopy group there were three such patients. Two had symptomatic trichomoniasis in addition to gonorrhoea, which was not diagnosed by microscopy but on culture. These two patients were not treated for their gonorrhoea, they did not attend their test of cure appointments for trichomoniasis and had given false names and addresses for contact tracing. One other patient treated for bacterial vaginosis from her microscopy results was finally traced for 
her positive culture for Neisseria gonorrhoeae, but by then had developed pelvic inflammatory disease and needed to attend the clinic for five subsequent appointments. Her contact was not found.

Omitting microscopy has potential disadvantages. There may be a delay in diagnosis. Does this matter? In this study one patient with gonorrhoea $(2.9 \%$ of cases, $0.1 \%$ of all samples) did not have microscopy. She was treated the next day when her partner attended with gonorrhoea.

Another drawback may arise if a patient defaults from follow-up and so fails to receive the results of culture. However, this did not occur in any patients with gonorrhoea in our study. Concern is often expressed that a delay in diagnosis means a potential to develop complications such as pelvic inflammatory disease. It is interesting to note that in the 1,000 women in this study only one patient developed pelvic inflammatory disease; she was in the universal microscopy group and had been diagnosed as having bacterial vaginosis alone at the first attendance.

Another worry is that patients without a definite diagnosis may unknowingly go on to infect other partners. There were no instances of this potential hazard in our selective microscopy group but two patients in the universal microscopy group treated only for Trichomonas vaginalis and then lost to followup may well have infected several other partners with their concurrent gonorrhoea.

There may also be a delay in the diagnosis of trichomoniasis with default from follow-up and potential transmission to new partners. Following the policy of performing microscopy if symptoms or discharge are present, all the patients with trichomoniasis had microscopy performed. Of the seven patients not diagnosed on microscopy in the selective microscopy group, two were lost to follow-up. One patient in the universal microscopy group had her trichomoniasis diagnosed by culture only. She attended for treatment, contact tracing and test of cure. It is again interesting to note that the false negative rate of microscopy for Trichomonas vaginalis in the universal microscopy group (one out of 16 or $6.25 \%$ ), is considerably lower than in the selective microscopy group (seven out of 17 or $43.75 \%$ ), despite a $28.6 \%$ reduction in workload.

Bacterial vaginosis and some cases of candidosis will obviously not be diagnosed if microscopy is not performed. As the policy of this department is not to treat these conditions if there are no symptoms, a policy of selective microscopy will have no effect on patient management in these cases. In this study, the incidence of gonorrhoea was $8 \%$ and trichomoniasis $3.5 \%$. The selective microscopy policy would be even more justifiable in clinics where the incidence of these conditions is lower.

A policy of selective microscopy amongst female patients attending genitourinary medicine clinics is therefore safe, effective and will lead to an increased efficiency of the service.

1 Rothenberg RB, Simon R, Chipperfield E, et al. Efficacy of selected diagnostic tests for sexually transmitted diseases. $\mathcal{F} A M A$ 1976;235:49-51

2 Barlow D, Phillips I. Gonorrhoea in women: Diagnostic and laboratory aspects. Lancet 1978;1:761-4.

3 Eschenback DA, Buchanan T, Pollock HM, et al. Polymicrobial etiology of acute PID. $N$ Eng $f \mathrm{Med}$ 1975;293:166-71.

4 Schmale JD, Morton JE Jnr, Domesciks S. Observation on the culture diagnosis of gonorrhoea in women. $f A M A$ 1969;210:312-4.

5 Thin RN, Shaw EJ. Diagnosis of gonorrhoea in women. Br F Venereal Dis 1979;55:10-3.

6 Barlow D, Nayyar K, Phillips I, Barron J. Diagnosis of gonorrhoea in women. Br ₹ Venereal Dis 1975;52:326-8. gonorrhoea in women. Br fenereal Dis 1975;52:326-8. 72:553-9.

8 Chipperfield EJ, Cotterall RD. Reappraisal of Gram staining and culture techniques for diagnosis of gonorrhoea in women. Br f Venereal Dis 1976;52:36-9.

9 Wald ER. Gonorrhoea-diagnosis by Gram stain in the female adolescent. Am $\mathcal{f}$ Dis Child 1977;131:1094-6.

10 Dans PE, Judson F. The establishment of a venereal disease clinic II: An appraisal of current diagnostic methods in uncomplicated urogenital and rectal gonorrhoea. 7 Am Venereal Dis Assoc 1975;1:107-12.

11 Whittington MJ. Epidemiology of infections with Trichomonas vaginalis in the light of improved diagnostic methods. Br F Venereal Dis 1957;3:380-91.

12 Acker JP, Lumsden WHR, Catterall RD, Coyle R Antitrichomonal antibody in the secretions of women infected with $T$ Vaginalis. Br $f$ Venereal Dis 1975;51: infected

13 Spence MR, Hollander DH, Smith JL, McCaig L, Sewell $\mathrm{D}$, Brockman M. The clinical and laboratory diagnosis of Trichomonas vaginalis infection. Sex Trans Dis 1980;7: of Trichom

14 Nagesha CN, Anantha Krishna NC, Swochana P. Clinical and laboratory studies on vaginal trichomonas. $\mathrm{Am} \mathcal{f}$ Obstet Gynecol 1970;106:933-4. 